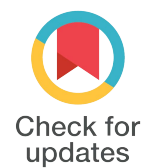

\section{Endovascular Repair of an Isolated Iliac Artery Aneurysm in a Patient with Liver Cirrhosis}

\author{
Azadeh Tahouni ${ }^{1}$, Milad Vahedinezhad ${ }^{1, *}$ \\ ${ }^{1}$ Cardiology Department, Iran University of Medical Sciences, Tehran, Iran \\ * Corresponding author: Milad Vahedinezhad, Cardiology Department, Iran University \\ of Medical Sciences, Tehran, Iran.E-mail:miladvahedinezhad@gmail.com
}

DOI: $10.29252 /$ ijcp-26616

\begin{tabular}{|c|c|}
\hline Submitted: 02-08-2019 & \multirow{7}{*}{$\begin{array}{l}\text { Abstract } \\
\text { Endovascular repair is increasingly becoming the main strategy for management of } \\
\text { aortic and peripheral aneurysms. Several methods have been introduced to treat } \\
\text { aneurysms based on anatomy, coexisting nearby aneurysms and available tools. We } \\
\text { presented a } 60 \text {-year-old man with an isolated large iliac artery aneurysm found } \\
\text { incidentally treated using an endovascular approach and obliterating internal iliac artery } \\
\text { without early and late complications at 3-year follow-up. }\end{array}$} \\
\hline Accepted: 09-08-2019 & \\
\hline Keywords: & \\
\hline Endovascular Procedures & \\
\hline Aneurysm & \\
\hline Arteries & \\
\hline $\begin{array}{l}\text { (C) 2019. International Journ } \\
\text { of Cardiovascular Practice. }\end{array}$ & \\
\hline
\end{tabular}

\section{INTRODUCTION}

Isolated iliac artery aneurysms (IIAA) are uncommon and mostly asymptomatic [1]. Endovascular aneurysm repair (EVAR) is the preferred method for therapeutic management of different types of aneurysms since mortality, morbidity, hospital stay and blood loss are less compared to surgical strategy and yet is as effective as open surgery [2]. Elective repair is indicated for common and external iliac artery aneurysms greater than 3-4 centimeters in maximal transverse diameter and 2 centimeters for internal iliac artery repair $[3,4]$. We reported here a case with large and isolated right common and external iliac artery aneurysm found incidentally during abdominal and pelvic computed tomography (CT) imaging.

\section{CASE REPORT}

A 60-year-old man was admitted with a history of liver cirrhosis to Firoozgar hospital, Tehran, Iran in 2016 due to complications of cirrhosis. On history taking, he complained about an intermittent claudication since several years ago, but was not his main compliant in the current admission. For evaluation of abdominal pain, he underwent an abdominal and pelvic CT with intravenous and oral contrast and a $7 \mathrm{~cm}$ sized right common and external iliac artery aneurysm was detected incidentally. Internal iliac artery was also minimally aneurysmal. Considering the indications mentioned above, we decided to repair the aneurysms. Because of the history of patient and potential risk for bleeding and decompensation of cirrhosis due to open surgery and also a favorable anatomy for endovascular approach, we decided to perform an EVAR.

Under general anesthesia bilateral femoral artery access was obtained by a vascular surgeon at angiography unit. Right iliac arteries angiogram is demonstrated in Figure 1. As shown, a large right common iliac artery aneurysm is seen on fluoroscopy. After catheterization, two Endurant TM stent grafts were deployed in common and external iliac arteries and for prevention of internal iliac artery endoleak considering the aneurysmal involvement of this vessel, two detachable Gold balloons were deployed in the right internal iliac artery. Final aortography showed complete closure of aneurysmal sac and no endoleak existed. Postoperative CT angiography is demonstrated in Figure 2 showing satisfactory results.

The procedure duration was about 2 hours. The patient's recovery course was uneventful and discharged from the interventional unit on the next day. $\mathrm{He}$ remained symptom-free without claudication in the follow-up visits and early or late complications of stent graft site did not occur after 3 years of follow up.

\section{DISCUSSION}

Our patient was admitted because of complications due to cirrhosis and his aneurysm caused no symptoms. However, repair of the aneurysm was indicated due to the size of the aneurysm and also the patient's underlying condition. Liver cirrhosis is independently associated with approximately $50 \%$ increased risk of 
postoperative complications in patients undergoing elective surgery and the main cause is increased bleeding tendency due to altered homeostatic balance [5]. In addition, because of iliac artery aneurysm's anatomic position deep in the pelvis and frequent presence of comorbidities, an open surgery is often challenging [6]. EVAR had become an alternative to treat IIAAs and proven to be safe and effective in several studies. In a systematic review and meta-analysis containing 7 studies which compared outcomes of open surgery and endovascular repair, Xiang et al. showed that both procedures were safe and effective to repair IIAAs with similar postoperative mortality. Compared with open surgery, endovascular treatment can reduce the need for blood transfusion and hospital stay, but it was associated with a higher risk of postoperative ischemic complications $[2,7]$.

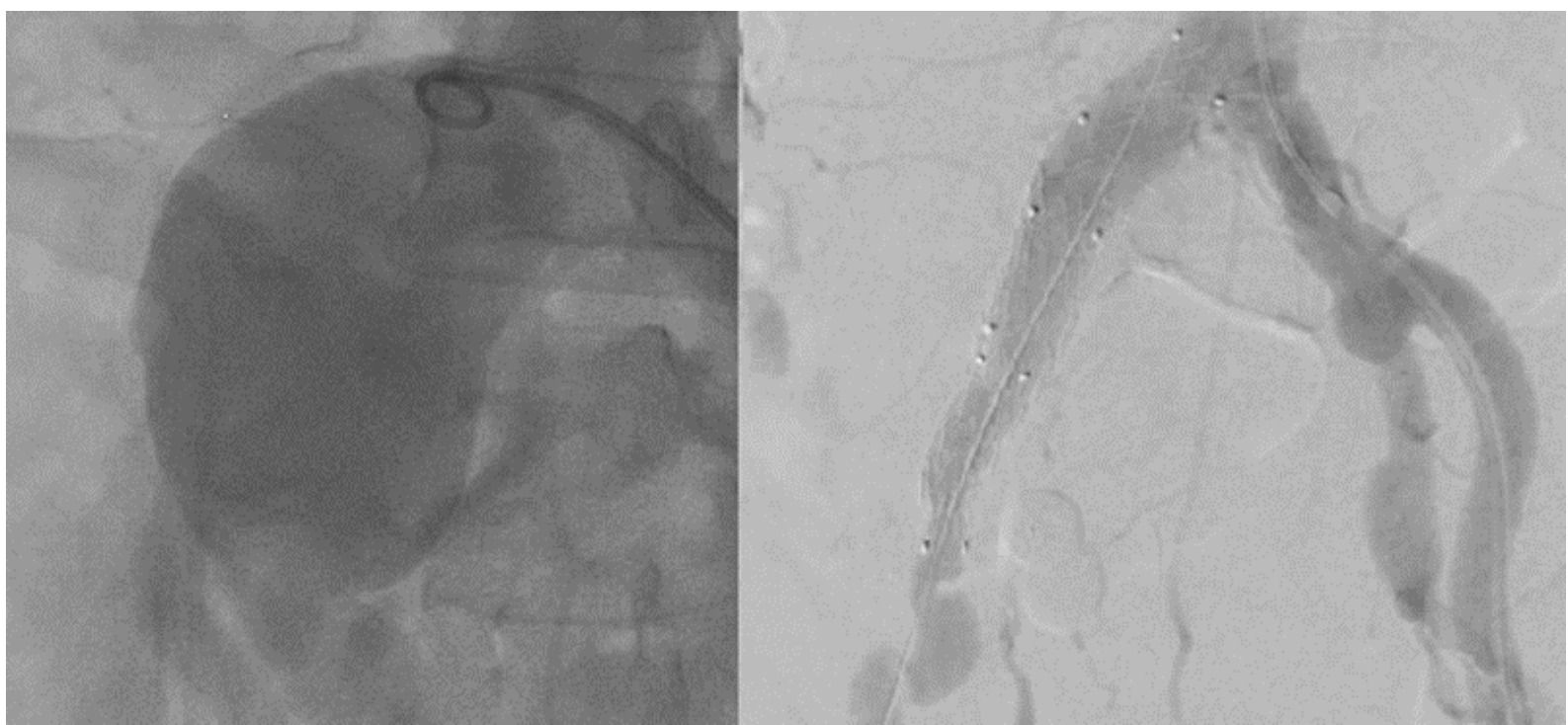

Figure 1. First Angiogram Showing a Large Common Iliac Aneurysm

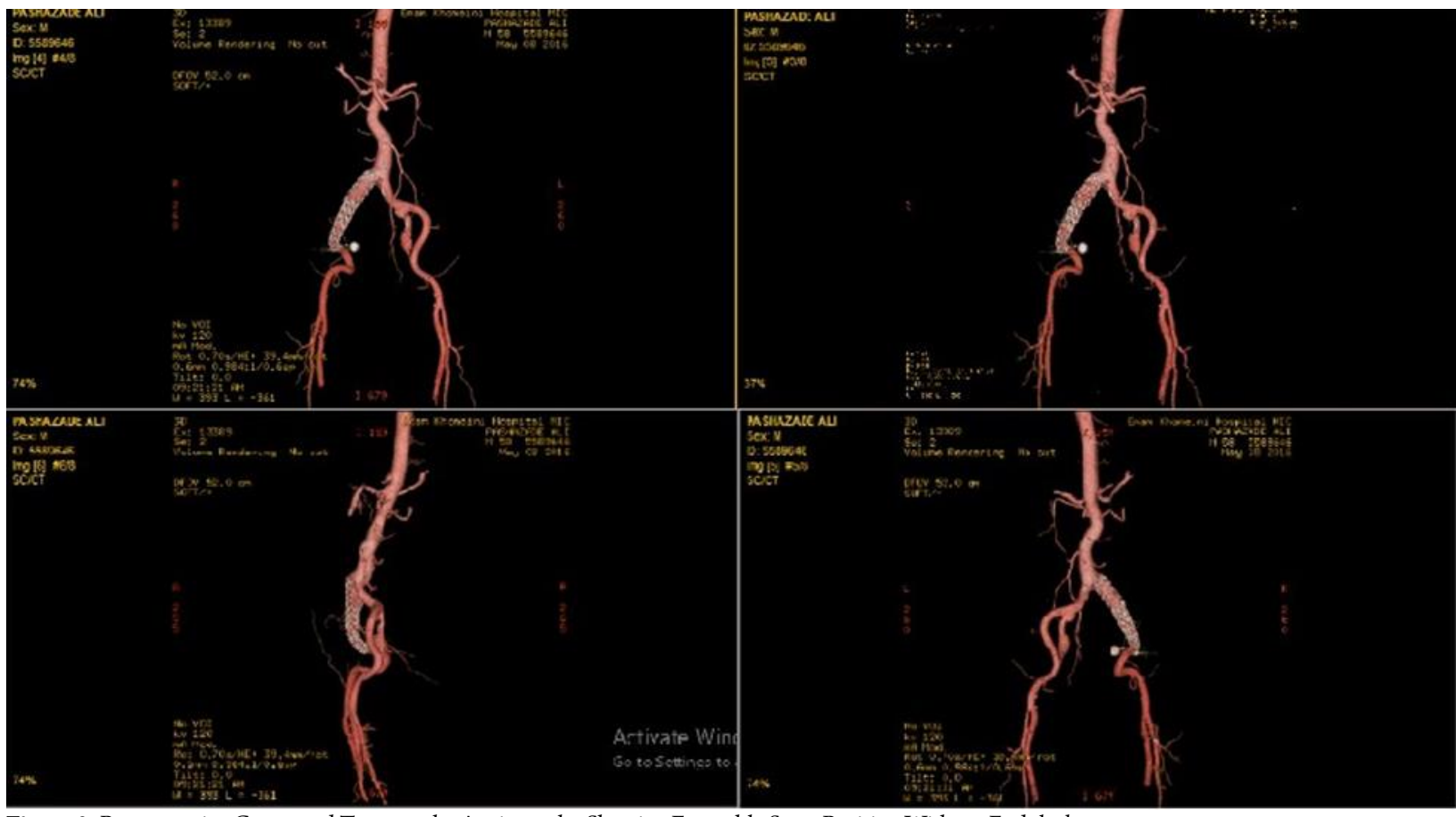

Figure 2. Postoperative Computed Topography Angiography Showing Favorable Stent Position Without Endoleak

We performed an endovascular repair of right common and external iliac arteries and obliteration of right hypogastric artery because of its aneurysmal involvement and prevention of endoleak. Recent studies have reported the importance of preservation of at least one hypogastric artery to reduce its postoperative complications. Hoo et al. demonstrated a new method using the AFXTM bifurcated device for common iliac artery distal fixation site to preserve internal iliac artery during common iliac artery aneurysm repair. However, 
in our case because of the special anatomy and involvement of distal portion of common iliac artery, preservation of right hypogastric artery was not possible. Because of intact anatomy of left hypogastric artery, complications due to obliteration of one hypogastric artery were not probable [8].

Our case never developed an endoleak which is a common complication of endovascular repair. Bryce et al. studied prevention and management of endoleaks and concluded that newer devices, such as the NellixTM endograft which consists of balloon-expandable stents with polymer-filled endobags, which fill the aneurysm sac may decrease the incidence of type II endoleaks because of its unique designs [7].

\section{Conflict of Interests}

None

\section{REFERENCES}

1. Richardson JW, Greenfield LJ. Natural history and management of iliac aneurysms. J Vasc Surg. 1988;8(2):16571. doi: $10.1067 / \mathrm{mva}$.1988.avs0080165 pmid: 3294450

2. Xiang Y, Chen X, Zhao J, Huang B, Yuan D, Yang Y. Endovascular Treatment Versus Open Surgery for Isolated Iliac Artery Aneurysms: A Systematic Review and Meta-
Analysis. Vasc Endovascular Surg. 2019;53(5):401-7. doi: 10.1177/1538574419836835 pmid: 30895896

3. Huang Y, Gloviczki P, Duncan AA, Kalra M, Hoskin TL, Oderich GS, et al. Common iliac artery aneurysm: expansion rate and results of open surgical and endovascular repair.J Vasc Surg. 2008;47(6):1203-10; discussion 10-1. doi: 10.1016/j.jvs.2008.01.050 pmid: 18514838

4. Kasirajan V, Hertzer NR, Beven EG, O'Hara PJ, Krajewski LP, Sullivan TM. Management of isolated common iliac artery aneurysms. Cardiovasc Surg. 1998;6(2):171-7. doi: 10.1016/S0967-2109(97)00136-1 pmid: 9610831

5. Biancofiore G, Blasi A, De Boer MT, Franchini M, Hartmann M, Lisman T, et al. Perioperative hemostatic management in the cirrhotic patient: a position paper on behalf of the Liver Intensive Care Group of Europe (LICAGE). Minerva Anestesiol. 2019;85(7):782-98. doi: 10.23736/S03759393.19.13468-2 pmid: 30945514

6. Cardon JM, Cardon A, Joyeux A, Vidal V, Noblet D. Endovascular repair of iliac artery aneurysm with Endoprosystem I: a multicentric French study. J Cardiovasc Surg (Torino). 1996;37(3 Suppl 1):45-50. pmid: 8707808

7. Bryce Y, Lam CK, Ganguli S, Schiro BJ, Cooper K, Cline M, et al. Step-by-Step Approach to Management of Type II Endoleaks. Tech Vasc Interv Radiol. 2018;21(3):188-95. doi: 10.1053/j.tvir.2018.06.009 pmid: 30497554

8. Hoo AS, Ryan L, Neville R, Mukherjee D. Customized endovascular repair of common iliac artery aneurysms. J Vasc Surg Cases Innov Tech. 2018;4(4):278-82. doi: 10.1016/j.jvscit.2018.07.009 pmid: 30547146 